\section{Asociación de los tipos de carencia y grado de desarrollo humano con la mortalidad infantil en México, 2008}

\author{
Association between types of need, human \\ development index, and infant mortality \\ in Mexico, 2008
}

Oswaldo Sinoe Medina-Gómez ${ }^{1}$

Oliva López-Arellano 2

\footnotetext{
1 Instituto Mexicano del Seguro Social, México DF, México.

2 Maestría en Medicina Social, Universidad Autónoma MetropolitanaXochimilco, Coyoacán, México.

Correspondencia O. S. Medina-Gómez Instituto Mexicano del Seguro Social. Arcoiris 66 Valle de luces Iztapalapa, Ciudad de México, DF 09800, México. epired@gmail.com
}

\section{Abstract}

The aim of this study was to assess the association between different types of economic and social deprivation and infant mortality rates reported in 2008 in Mexico. We conducted an ecological study analyzing the correlation and relative risk between the human development index and levels of social and economic differences in State and national infant mortality rates. There was a strong correlation between higher human development and lower infant mortality. Low schooling and poor housing and crowding were associated with higher infant mortality. Although infant mortality has declined dramatically in Mexico over the last 28 years, the decrease has not been homogeneous, and there are persistent inequalities that determine mortality rates in relation to different poverty levels. Programs with a multidisciplinary approach are needed to decrease infant mortality rates through comprehensive individual and family development.

Health Inequalities; Infant Mortality; Poverty

\section{Introducción}

A pesar de que se han hecho esfuerzos importantes en materia de salud pública 1, el incremento de las desigualdades e inequidades en salud en América Latina continúa hasta nuestros días. Recientemente, se ha consolidado un creciente interés sobre los determinantes sociales de la salud para comprender las inequidades en salud y brindar nuevas opciones de solución 2,3,4,5,6,7,8.

La mortalidad infantil forma parte de los daños a la salud que deben ser afrontados por los países. Su reducción está prevista entre los compromisos que las naciones del mundo han suscrito dentro del marco de los Objetivos de Desarrollo del Milenio. Sin embargo, en la región de América Latina se presenta una gran heterogeneidad entre los países, mientras Cuba y Chile reportan tasas de mortalidad infantil de 4,7 y 8,3 por 1.000 nacidos vivos respectivamente, en Haití se alcanzan tasas de 57,0 muertes por 1.000 nacidos vivos 9,10 .

La mortalidad infantil es reconocida como un indicador que expresa muertes evitables, innecesarias y prematuras, que pueden prevenirse mediante acciones individuales y colectivas de atención, e intervenciones sociales orientadas a influir sobre los determinantes sociales y económicos 11,12. Estas muertes son un referente que expresan el grado de inequidad en salud de una población, por ello, los diferenciales en su frecuencia no reflejan únicamente la presencia de 
desigualdades en la atención médico-sanitaria, sino que también apuntan a las desigualdades e injusticias estructurales y a la necesidad de promover políticas públicas saludables encaminadas a modificar los determinantes sociales que les dan origen 13 .

El análisis de las condiciones, que determinan desigualdades en la mortalidad infantil en el país, debe abordarse desde distintos planos, destacando los determinantes sociales expresados como marginación, pobreza, ruralidad y falta de acceso a satisfactores esenciales como educación y salud, que en diversos estudios han mostrado históricamente una estrecha relación con incrementos en las tasas de mortalidad infantil 14,15,16,17,18,19.

Por lo anterior, el objetivo del presente estudio fue conocer la asociación entre los diferentes tipos de carencia social y económica y los niveles de mortalidad infantil reportados durante el 2008 en México.

\section{Material y métodos}

Se realizó un estudio ecológico, a partir de fuentes secundarias de información correspondientes al año 2008, de las 32 entidades federativas que conforman México. El Sistema de Información en Salud de la Secretaría de Salud de México fue la fuente de información para conocer la tasa de mortalidad infantil (TMI) a nivel estatal. La tasa de mortalidad infantil se definió como el número de defunciones que ocurren de menores de un año, entre el número de nacidos vivos en un año determinado, según los nacimientos estimados por el Consejo Nacional de Población (CONAPO). Para conocer el índice de pobreza, así como sus componentes, se consultaron los reportes emitidos por el Consejo Nacional de Evaluación (CONEVAL). Para identificar el nivel de escolaridad del jefe de familia, se utilizó la información emitida por el Conteo Nacional de Vivienda 2005 del Instituto Nacional de Estadística y Geografía (INEGI), dado que es la única fuente oficial cercana al año bajo estudio, finalmente se utilizó el índice de desarrollo humano reportado por la Organización de las Naciones Unidas (ONU) 20.

Se utilizó el paquete estadístico Stata versión 9.0 (Stata Corp., College Station, EE.UU.) para el análisis de los datos obtenidos, el análisis descriptivo se realizó por cada indicador de atraso de cada entidad federativa. Se analizó la relación entre la TMI y los indicadores de carencia social y económica por quintiles, mediante los coeficientes de correlación de Spearman.

Mediante el programa SIGEpi versión 1.26 (Organización Panamericana de la Salud; http:// ais.paho.org/sigepi/index.asp) se construyó un mapa de las entidades federales con la distribución geográfica de la tasa de mortalidad clasificada en cuartiles, con el fin de facilitar la identificación territorial del problema de salud de interés.

Finalmente, se ajustaron modelos de regresión binomial negativa generalizada para calcular el riesgo relativo (RR) de mortalidad, según los indicadores carenciales, teniendo como variable dependiente la TMI y como independientes cada uno de los indicadores carenciales clasificados por quintiles, el número de nacidos vivos sirvió como constante para el modelo.

\section{Resultados}

La población total de México estimada por CONAPO en el 2008 era de 105.790 .725 habitantes, de los cuales $1.934 .349(1,83 \%)$ eran menores de 1 año (988.844 hombres y 945.505 mujeres). De acuerdo a las cifras registradas, la mortalidad infantil a nivel nacional en 1990 fue de 39,2 muertes por cada 1.000 nacidos vivos, mientras que durante el 2008 fue de 15,2 por cada 1.000 nacidos vivos, la distribución de las mayores tasas de mortalidad infantil se concentran principalmente en los estados del sur del país (Figura 1).

Para el año 2008 el 43,8\% de las entidades federativas presentaron cifras por arriba de la tasa nacional y se encontró que la disminución en las tasas de mortalidad infantil se distribuye de manera diferenciada entre los estados de la república, mientras el estado de Nuevo León reporta una tasa de 10,6 por cada 1.000 nacidos vivos, estados como Chiapas y Guerrero muestran una tasa de alrededor de 21 por cada 1.000 nacidos vivos. A nivel nacional se ha reducido la tasa de mortalidad infantil en un $0,5 \%$ con un rango de $0,3 \%$ a $0,7 \%$ (Figura 2 ).

El análisis de regresión lineal simple mostró una correlación importante $\left(\mathrm{R}^{2}=0,67 ; \mathrm{p}<0,001\right)$ entre la tasa de mortalidad infantil con el grado de desarrollo humano reportado por la ONU.

El porcentaje de hogares con jefes de familia sin escolaridad o escolaridad menor a primaria, en comparación con los hogares cuyos jefes de familia cuentan con licenciatura, los resultados mostraron que existe una fuerte correlación entre un bajo grado de escolaridad de los jefes de familia y el incremento de mortalidad infantil $\left(\mathrm{R}^{2}=0,83 ; \mathrm{p}<0,001\right)$ (Figura 3).

También se encontró una correlación estadísticamente significativa al nivel de $1 \%$ entre la mortalidad infantil y cada uno de los componentes del índice de pobreza, siendo que la mayor asociación encontrada es con la carencia por acceso a alimentación $\left(\mathrm{R}^{2}=0,625\right.$; $\left.\mathrm{p}<0,001\right)$ 
Figura 1

Distribución de la tasa de mortalidad según entidad federativa de México, 2008.

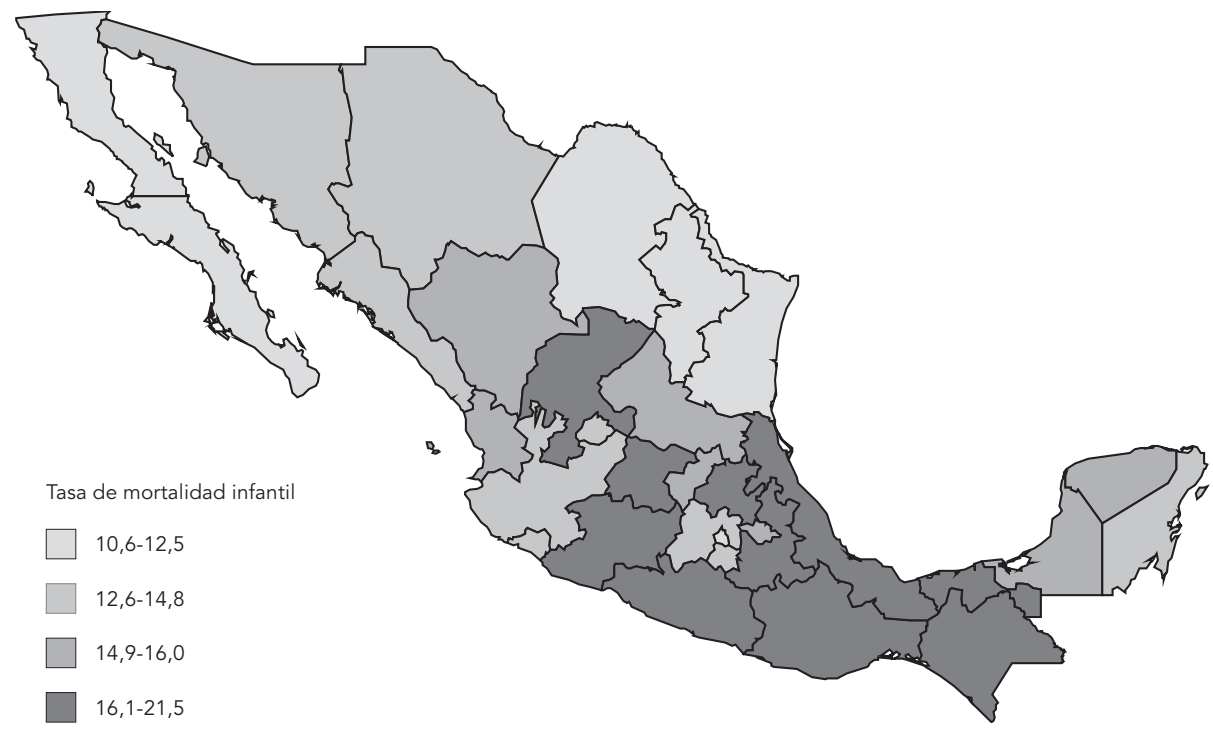

Figura 2

Porcentaje de disminución de la mortalidad infantil según las entidades federativas de México, 2007 al 2008.

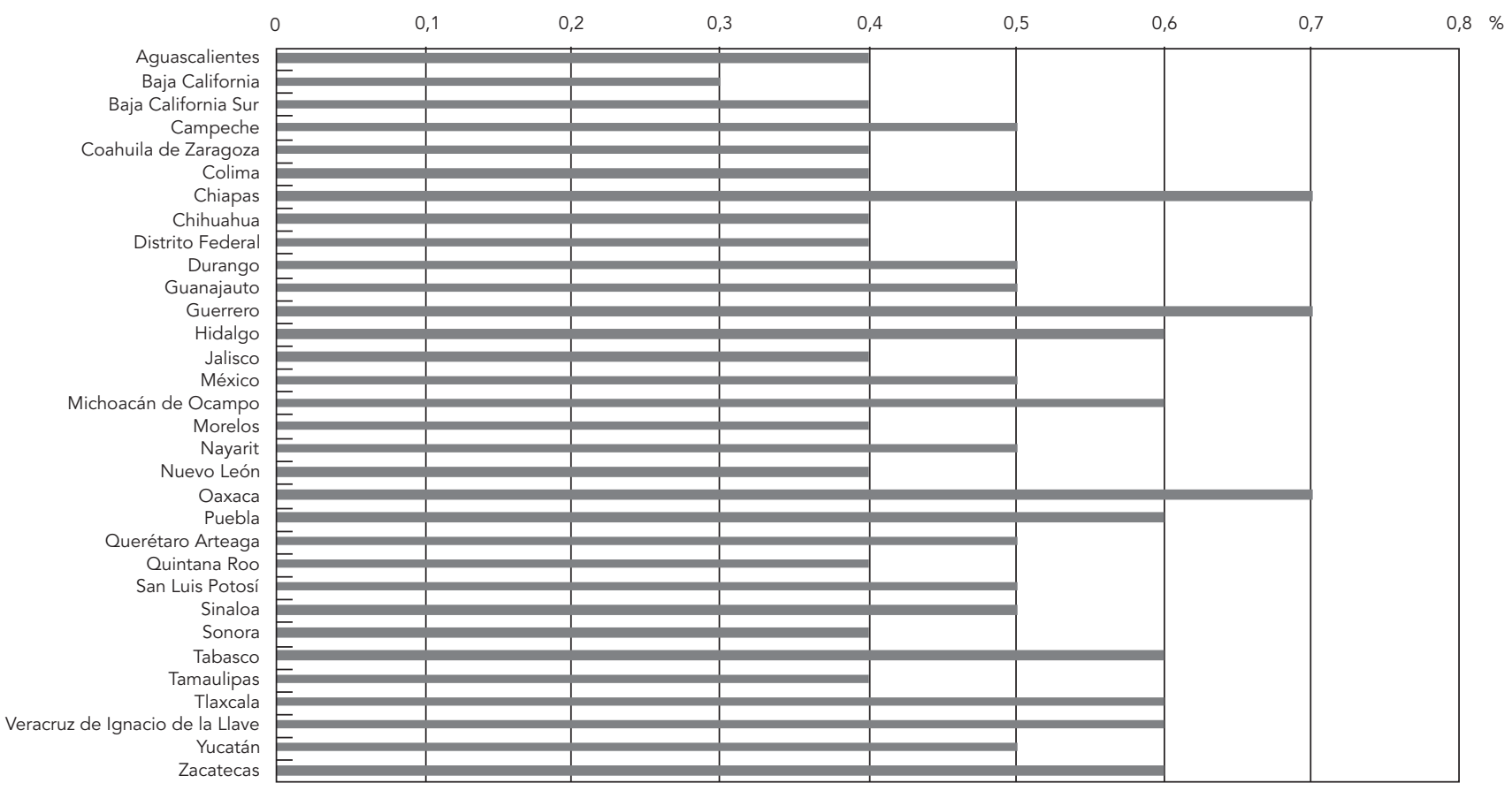




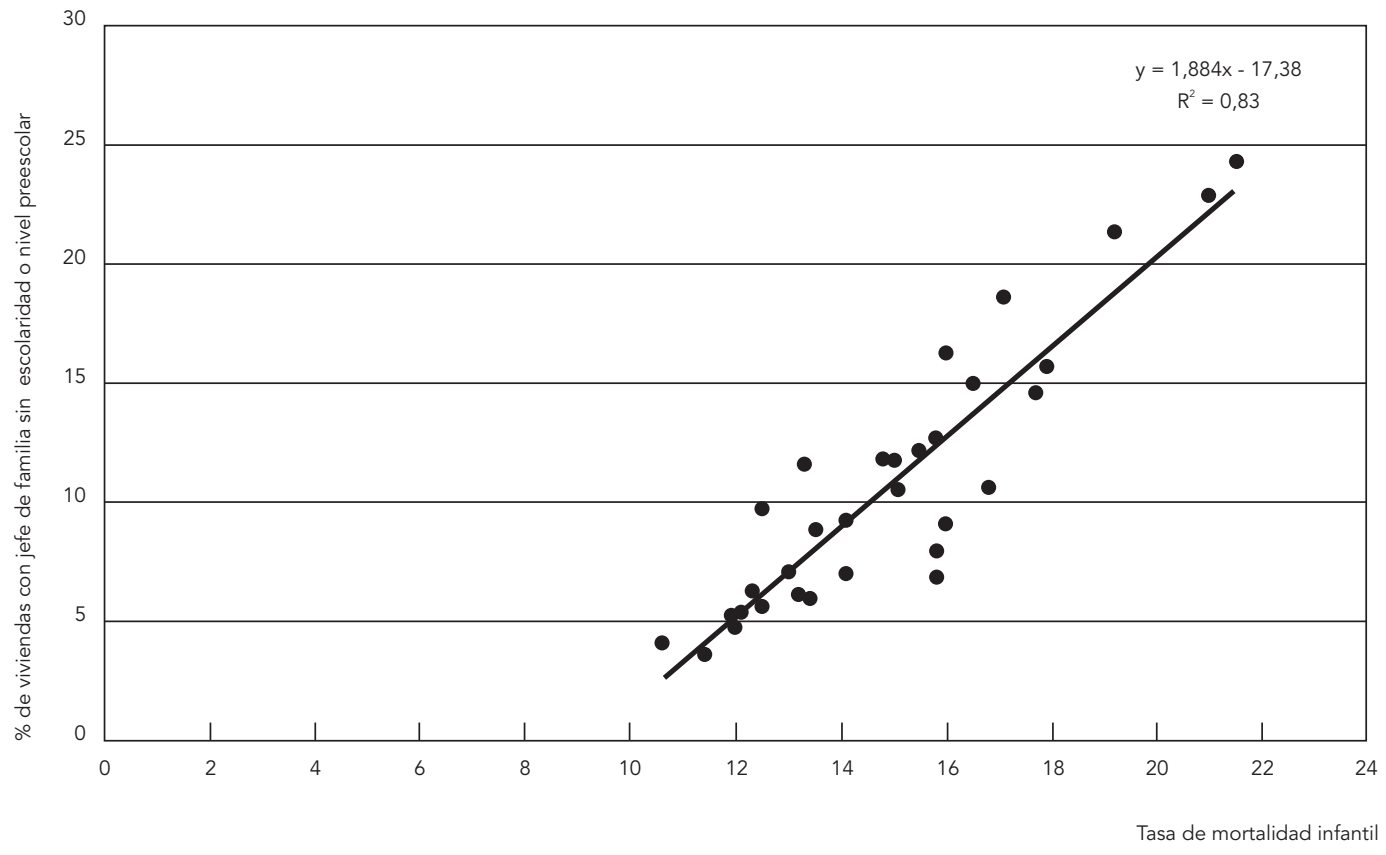

y la menor correlación es con el atraso educativo $(\mathrm{R}=0,427 ; \mathrm{p}=0,015)$ (Tabla 1$)$.

Cuando se compara mediante la regresión binomial negativa generalizada la TMI con los componentes del índice de pobreza agrupados en quintiles, se observó que a mayor atraso educativo de la población el riesgo de mortalidad infantil es mayor ( $R R=7,47$; IC95\%: 1,54-36,33). De igual manera, las entidades federativas con atraso en la calidad y espacios de la vivienda ubicados en el quintil 4 muestran un mayor riesgo de mortalidad infantil ( $R R=2,57$; IC95\%: 1.17 5.61) (Tabla 2).

\section{Discusión}

Derivado del tipo de estudio realizado, debe considerarse la posibilidad de la falacia ecológica sin por ello demeritar los resultados obtenidos. El uso de fuentes secundarias de información se encuentra limitado, debido a que fueron creadas para fines específicos distintos a los objetivos de la presente investigación, por lo que la información recuperada de registros y bases de datos no siempre responde a las necesidades para lo que se les utiliza 21 .

Por eso resulta necesario resaltar la necesidad de contar con información que permita no sólo conocer los niveles de pobreza multidimensional, sino que reconozca las condiciones de vida de las personas que viven en condiciones de pobreza, dado que las definiciones de cada componente carencial identifican la presencia de la carencia de al menos un servicio o característica. Tal es el caso del atraso en el acceso a la salud, puesto que identifica sólo la falta de afiliación a una institución o programa público de salud, sin dar cuenta del nivel de acceso y disponibilidad de los servicios de salud por parte de la población.

El comportamiento de la mortalidad infantil en México en 2008 y las asociaciones entre grado de desarrollo humano, marginación, pobreza y escolaridad identificadas en este trabajo son consistentes con lo reportado en la literatura científica sobre los determinantes sociales de la mortalidad infantil 19,22,23,24,25,26,27. Al sistematizar los datos a nivel de entidades federativas, se avanza en superar el indicador nacional, que bajo su medición por promedio se enmascaran 
Análisis de la correlación entre los componentes del índice de pobreza y la tasa de mortalidad infantil. México, 2008.

\begin{tabular}{|c|c|c|c|c|c|c|c|c|}
\hline Tipo de carencia & & $\begin{array}{c}\text { Atraso } \\
\text { educativo }\end{array}$ & $\begin{array}{l}\text { Acceso a } \\
\text { servicios } \\
\text { de salud }\end{array}$ & $\begin{array}{l}\text { Acceso a } \\
\text { seguridad } \\
\text { social }\end{array}$ & $\begin{array}{l}\text { Calidad y } \\
\text { espacios } \\
\text { de la } \\
\text { vivienda }\end{array}$ & $\begin{array}{l}\text { Servicios } \\
\text { básicos } \\
\text { en la } \\
\text { vivienda }\end{array}$ & $\begin{array}{c}\text { Acceso a } \\
\text { alimentación }\end{array}$ & $\begin{array}{c}\text { Tasa de } \\
\text { mortalidad } \\
\text { infantil } 2008\end{array}$ \\
\hline Atraso educativo & $\begin{array}{l}\text { Correlación } \\
\text { Significancia }\end{array}$ & 1 & & & & & & \\
\hline $\begin{array}{l}\text { Acceso a servicios de } \\
\text { salud }\end{array}$ & $\begin{array}{l}\text { Correlación } \\
\text { Significancia }\end{array}$ & $\begin{array}{c}0,616 * \\
0\end{array}$ & 1 & & & & & \\
\hline Acceso a seguridad social & $\begin{array}{l}\text { Correlación } \\
\text { Significancia }\end{array}$ & $\begin{array}{c}0,801 * \\
0\end{array}$ & $\begin{array}{c}0,757 * \\
0\end{array}$ & 1 & & & & \\
\hline $\begin{array}{l}\text { Calidad y espacios de la } \\
\text { vivienda }\end{array}$ & $\begin{array}{l}\text { Correlación } \\
\text { Significancia }\end{array}$ & $\begin{array}{c}0,779 * \\
0\end{array}$ & $\begin{array}{c}0,623 * \\
0\end{array}$ & $\begin{array}{c}0,706 \text { * } \\
0\end{array}$ & 1 & & & \\
\hline $\begin{array}{l}\text { Servicios básicos en la } \\
\text { vivienda }\end{array}$ & $\begin{array}{l}\text { Correlación } \\
\text { Significancia }\end{array}$ & $\begin{array}{c}0,803 * \\
0\end{array}$ & $\begin{array}{c}0,654 \text { * } \\
0\end{array}$ & $\begin{array}{c}0,733 * \\
0\end{array}$ & $\begin{array}{c}0,897 \text { * } \\
0\end{array}$ & 1 & & \\
\hline Acceso a alimentación & $\begin{array}{l}\text { Correlación } \\
\text { Significancia }\end{array}$ & $\begin{array}{c}0,657 \text { * } \\
0\end{array}$ & $\begin{array}{c}0,631 \text { * } \\
0\end{array}$ & $\begin{array}{c}0,821 * \\
0\end{array}$ & $\begin{array}{c}0,589 \text { * } \\
0\end{array}$ & $\begin{array}{c}0,671 \text { * } \\
0\end{array}$ & 1 & \\
\hline $\begin{array}{l}\text { Tasa de mortalidad } \\
\text { infantil } 2008\end{array}$ & $\begin{array}{l}\text { Correlación } \\
\text { Significancia }\end{array}$ & $\begin{array}{c}0,427 * * \\
0,015\end{array}$ & $\begin{array}{c}0,429 * * \\
0,014\end{array}$ & $\begin{array}{c}0,446 \text { ** } \\
0,011\end{array}$ & $\begin{array}{c}0,451 * \\
0,01\end{array}$ & $\begin{array}{c}0,579 * \\
0,001\end{array}$ & $\begin{array}{c}0,625 * \\
0\end{array}$ & 1 \\
\hline
\end{tabular}

* La correlación es significativa en el nivel 0,01 (bilateral);

** La correlación es significativa en el nivel 0,05 (bilateral).

las inequidades locales. Así, en este trabajo se reconocen algunos de los determinantes sociales, que al ser analizados en su dimensión estatal identifican consistentemente situaciones diferenciadas de desarrollo, pobreza y marginación. Estos determinantes se corresponden con desigualdades en las tasas de mortalidad infantil y se expresan como brechas interestatales.

Las tasas de mortalidad a nivel nacional muestran una tendencia hacia la baja, aún bajo la acción de políticas focalizadas que incluyen acciones específicas para disminuir la mortalidad infantil. Sin embargo, es evidente que cuando observamos las tasas específicas por cada entidad federativa se expresa una enorme desigualdad en el indicador seleccionado. Si bien se ha presentado una disminución en la mortalidad infantil en todos los estados, al mantener una disminución similar entre ellos, no se logra acortar la brecha de inequidad existente entre los estados más pobres que presentan las tasas de mortalidad infantil más elevadas, en relación con los estados más desarrollados. Las tasas de mortalidad infantil son tan desiguales, lo cual explica por qué estados con elevada marginación como Chiapas, presenta en 2008 tasas de mortalidad infantil similares a la reportada en la tasa nacional de 1999. Sin embargo, resulta importante realizar estudios posteriores que ex- ploren las características de la población tales como la etnia, procesos migratorios, características de la familia, acceso geográfico, barreras culturales y su relación con las tasas de mortalidad infantil.

Para obtener un mayor beneficio en salud y un efecto positivo sobre la mortalidad infantil, es necesario aplicar políticas saludables que trasciendan el ámbito sectorial, por ejemplo, garantizar el acceso a la educación y la universalización en el acceso a los servicios de salud. Si se reconoce que el bajo nivel escolar del jefe de familia se asocia a una mayor tasa de mortalidad infantil, este campo tendría que ser prioritario como parte del proceso de desarrollo de las personas y las comunidades. La promoción de la salud debería considerar otros componentes como la defensa y preservación del medio ambiente, la seguridad nutricional y fortalecer todas aquellas dimensiones que conllevan una mejor vida de los individuos y sus comunidades, que finalmente se traducen en bienestar y salud 28,29.

Sin duda para quienes diseñan e implementan políticas en salud, es imprescindible aproximarse al proceso salud-enfermedad con una mirada transdisciplinaria que permita conocer cada una de sus facetas, así como la interacción entre las distintas dimensiones biológicas y sociales del proceso. 
Asociación entre la tasa de mortalidad infantil y los indicadores de atraso. México, 2008.

\begin{tabular}{|c|c|c|c|c|}
\hline Tipo de atraso por quintiles & RR & Valor de $\mathrm{p}$ & \multicolumn{2}{|c|}{ IC95\% } \\
\hline 1 & 1 & & & \\
\hline 2 & 1,621412 & 0,303 & 0,6468194 & 4,064469 \\
\hline 3 & 4,786257 & 0,002 & 1,787718 & 12,81424 \\
\hline 4 & 6,04487 & 0,006 & 1,680836 & 21,73946 \\
\hline 5 & 7,470357 & 0,013 & 1,535953 & 36,33329 \\
\hline \multicolumn{5}{|c|}{ Carencia por acceso a servicios de salud } \\
\hline 1 & 1 & & & \\
\hline 2 & 0,7708444 & 0,519 & 0,3491795 & 1,701707 \\
\hline 3 & 0,260088 & 0,001 & 0,1139167 & 0,5938178 \\
\hline 4 & 0,3041659 & 0,001 & 0,1551852 & 0,5961709 \\
\hline 5 & 0,3527254 & 0,031 & 0,1370997 & 0,9074802 \\
\hline \multicolumn{5}{|c|}{ Carencia por acceso a seguridad social } \\
\hline 1 & 1 & & & \\
\hline 2 & 0,5674124 & 0,231 & 0,2245404 & 1,433848 \\
\hline 3 & 1,703273 & 0,292 & 0,6328921 & 4,583938 \\
\hline 4 & 1,577873 & 0,374 & 0,5772823 & 4,312766 \\
\hline 5 & 2,040278 & 0,223 & 0,6484162 & 6,419847 \\
\hline \multicolumn{5}{|c|}{ Carencia por calidad y espacios de la vivienda } \\
\hline 1 & 1 & & & \\
\hline 2 & 5,153136 & 0 & 2,885684 & 9,202257 \\
\hline 3 & 1,834857 & 0,105 & 0,8807534 & 3,822523 \\
\hline 4 & 2,565905 & 0,018 & 1,173135 & 5,612199 \\
\hline 5 & 2,354555 & 0,060 & 0,9648275 & 5,746032 \\
\hline \multicolumn{5}{|c|}{ Carencia por servicios básicos en la vivienda } \\
\hline 1 & 1 & & & \\
\hline 2 & 0,776959 & 0,502 & 0,371817 & 1,623555 \\
\hline 3 & 0,1482327 & $<0,001$ & 0,059644 & 0,3684016 \\
\hline 4 & 0,0954515 & $<0,001$ & 0,0347432 & 0,2622382 \\
\hline 5 & 0,062314 & $<0,001$ & 0,0199786 & 0,19436 \\
\hline \multicolumn{5}{|c|}{ Carencia por acceso a alimentación } \\
\hline 1 & 1 & & & \\
\hline 2 & 0,6806271 & 0,159 & 0,3982729 & 1,163155 \\
\hline 3 & 0,9246977 & 0,831 & 0,4506477 & 1,897416 \\
\hline 4 & 0,9742018 & 0,945 & 0,4627792 & 2,050803 \\
\hline 5 & 0,7136754 & 0,398 & 0,3266025 & 1,559488 \\
\hline
\end{tabular}

IC95\%: intervalo de 95\% de confianza; RR: riesgo relativo.

Es importante que los datos permitan reconocer procesos y que las expresiones numéricas sean indicativas de los contextos donde se presentan los problemas y hagan visibles las desigualdades socio-sanitarias. En este sentido, el análisis presentado en este trabajo permite destacar las diferencias entre los estados y su relación con la mortalidad infantil.

El reconocimiento de los determinantes sociales de la salud, entre los que destacan la po- breza y la marginación y su relación con los daños a la salud 27 , en este caso con la mortalidad infantil, amplía la perspectiva de acción colectiva y alimenta las propuestas para el abordaje integral de éste y otros problemas sanitarios. 


\section{Resumen}

El objetivo del artículo fue conocer la asociación entre los diferentes tipos de carencia social y económica y los niveles de mortalidad infantil reportados durante el 2008 en México. Se realizó un estudio ecológico, analizando la correlación y el riesgo relativo entre el índice de desarrollo humano y distintos niveles de carencias sociales y económicas con las tasas de mortalidad infantil reportadas a nivel nacional y estatal. Existe una fuerte correlación entre un mayor nivel de desarrollo humano con una menor tasa de mortalidad. La carencia educativa y el atraso en la calidad y espacio de la vivienda se asocian con una mayor tasa de mortalidad infantil. Si bien la mortalidad infantil en México ha disminuido notablemente en los últimos 28 años, su reducción no ha sido homogénea y se mantienen inequidades que determinan las tasas de mortalidad en relación a los niveles diferenciados de pobreza. Es necesario el diseño de programas con una visión transdisciplinaria que permitan disminuir las tasas de mortalidad con el pleno desarrollo de los individuos y sus familias

Desigualdades en la Salud; Mortalidad Infantil; Pobreza

\section{Colaboradores}

O. S. Medina-Gómez contribuyó con el diseño teórico, metodológico y análisis. O. López-Arellano contribuyó con el diseño teórico del proyecto. Los autores escribieron el artículo.

\section{Referencias}

1. Sepúlveda J, Bustreo F, Tapia R, Rivera J, Lozano $\mathrm{R}$, Olaiz G, et al. Improvement of child survival in Mexico: the diagonal approach. Lancet 2006; 368:2017-27.

2. Paim JP, Almeida Filho N. A crise da saúde pública, a utopia da saúde coletiva. Salvador: Casa da Qualidade Editora; 2000.

3. Starfield B. Equity and health: a perspective on nonrandom distribution of health in the population. Rev Panam Salud Pública 2002; 12:384-7.

4. Alazraqui M, Mota E, Spinelli H, Guevel C. Inequalities in health and socioeconomics: an epidemiological undertaking in an urban area of Argentina. Rev Panam Salud Pública 2007; 21:1-10.
5. Almeida-Filho N. La ciencia tímida. Ensayos de deconstrucción de la epidemiología. Buenos Aires: Lugar Editorial; 2000.

6. Bacallao J, Castillo-Salgado C, Schneider MC, Mujica OJ, Loyola E, Vidaurre M. Indices based on entropy for measuring social inequalities in health. Rev Panam Salud Pública 2002; 12:429-35.

7. Pearce N. The globalization of epidemiology: introductory remarks. Int J Epidemiol 2004; 33:1127-31.

8. Barreto ML. The globalization of epidemiology: critical thoughts from Latin America. Int J Epidemiol 2004; 33:1132-7.

9. Bahr J, Wehrhahn R. Life expectancy and infant mortality in Latin America. Soc Sci Med 1993; 36: 1373-82. 
10. Pan American Health Organization. Health information and analysis project. Health situation in the Americas: basic indicators 2009. Washington DC: Pan American Health Organization; 2009.

11. Whitehead M. The concepts and principles of equity and health. Int J Health Serv 1992; 22:429-45.

12. World Health Organization. Equity in health and health care: a WHO/SIDA initiative. Geneva: World Health Organization; 1996.

13. Braveman P, Starfield B, Geiger HJ. World Health Report 2000: how it removes equity from the agenda for public health monitoring and policy. BMJ 2001; 323:678-81.

14. Matteson DW, Burr JA, Marshall JR. Infant mortality: a multi-level analysis of individual and community risk factors. Soc Sci Med 1998; 47:1841-54.

15. Aksit B. Sociocultural determinants of infant and child mortality in Turkey. Soc Sci Med 1989; 28:571-6.

16. Lin CC, Kuo PH, Su CH, Chen WJ. The Taipei Adolescent Twin/sibling Family Study I: behavioral problems, personality features, and neuropsychological performance. Twin Res Hum Genet 2006; 9:890-4.

17. Kunitz SJ, Simic S, Odoroff CL. Infant mortality and economic instability in Yugoslavia. Soc Sci Med 1987; 24:953-60.

18. Antonovsky A, Bernstein J. Social class and infant mortality. Soc Sci Med 1977; 11:453-70.

19. Frank R, Finch BK. Los años de la crisis: an examination of change in differential infant mortality risk within Mexico. Soc Sci Med 2004; 59:825-35.

20. Lemaresquier T, Peral T. Informe sobre el desarrollo humano en México 2006-2007. México DF: Programa de las Naciones Unidas para el Desarrollo; 2007.
21. Borja-Aburto VH. Estudios ecológicos. Salud Pública Méx 2000; 42:533-8.

22. Menezes AM, Hallal PC, Santos IS, Victora CG, Barros FC. Infant mortality in Pelotas, Brazil: a comparison of risk factors in two birth cohorts. Rev Panam Salud Pública 2005; 18:439-46.

23. Maydana E, Serral G, Borrell C. Socioeconomic inequalities and infant mortality in Bolivia. Rev Panam Salud Pública 2009; 25:401-10.

24. Gattini C, Sanderson C, Castillo-Salgado C. Using different indicators of preventable mortality as an approach to measuring health inequalities in Chilean municipalities. Rev Panam Salud Pública 2002; 12:454-61.

25. Mayer SE, Sarin A. Some mechanisms linking economic inequality and infant mortality. Soc Sci Med 2005; 60:439-55.

26. Gakidou E, Cowling K, Lozano R, Murray CJ. Increased educational attainment and its effect on child mortality in 175 countries between 1970 and 2009: a systematic analysis. Lancet 2010; 376: 959-74.

27. Rodríguez-García J. Socioeconomic inequality and its association with mortality indicators in the departments of Colombia in 2000. Rev Panam Salud Pública 2007; 21:111-24.

28. Sen A. La salud en el desarrollo: variables socioeconómicas en el análisis de la situación de salud. Managua: Centro de Investigaciones y Estudios de la Salud; 2005.

29. Orellana D. La salud en la globalización. Quito: Centro sobre Derecho y Sociedad; 2003.

Recibido el 18/Ene/2011

Versión final presentada el 16/May/2011 Aprobado el 07/Jun/2011 